\title{
Estrutura e desempenho da assistência médica e odontológica em duas regiões de saúde brasileiras entre 2007 e 2014
}

\author{
Structure and performance of medical and dental care \\ in two Brazilian health regions between 2007 and 2014
}

Joana Danielle Brandão Carneiro (https://orcid.org/0000-0003-3629-8091) ${ }^{1}$

Aylene Bousquat (https://orcid.org/0000-0003-2701-1570) ${ }^{1}$

Sônia Cristina Lima Chaves (https://orcid.org/0000-0002-1476-8649) ${ }^{2}$

Paulo Frazão (http://orcid.org/0000-0002-3224-0020) ${ }^{1}$

${ }^{1}$ Faculdade de Saúde Pública, Universidade de São Paulo. Av. Dr. Arnaldo 715, Cerqueira César. 01246904 São Paulo SP Brasil. joanadani9@gmail.com ${ }^{2}$ Instituto de Saúde Coletiva, Universidade Federal da Bahia. Salvador BA Brasil.

\begin{abstract}
The structure and performance of medical and dental care were analyzed in two health regions that differed socioeconomically and in the provision of services, through case study in the Norte-Barretos (São Paulo) and Juazeiro (Bahia) regions from 2007 and 2014, taking into account political, organizational and structural dimensions and structure and performance indicators. The results showed that the regionalization was positively recognized, the distribution of services did not meet the population demand, and the installed capacity of the health care network was not adequate for the health needs of the population. Norte-Barretos stood out regarding structure (except for potential coverage of oral health teams in the Family Health Strategy) and effectiveness, while Juazeiro stood out concerning efficiency; e.g. although with fewer resources, the use of services was relatively higher. The observed pattern seems to reflect aspects related to the regionalization and the political path of each care provided, the socalled "silos effect". The results may support the design of health policies aimed at overcoming the undersized structure of public health services in regions of lower socioeconomic development and search for parameters and coordination mechanisms to balance performance indicators better.

Key words Health policy, Regionalization, Dental care, Comprehensive healthcare
\end{abstract}

Resumo O artigo analisa a estrutura e o desempenho das assistências médica e odontológica de duas regiões, distintas socioeconomicamente $e$ na oferta de serviços, por meio de estudo de caso nas regiões Norte-Barretos (São Paulo) e Juazeiro (Bahia) entre 2007-2014, a partir das dimensões politica, organizacional e estrutural e de indicadores de estrutura e desempenho. Observou-se que a regionalização foi reconhecida de forma positiva, a distribuição dos serviços não atendia à demanda populacional e a capacidade instalada da rede de atenção à saúde não estava adequada às necessidades da população. Norte-Barretos se sobressaiu em relação à estrutura (exceto para cobertura potencial da ESB na ESF) e efetividade; enquanto Juazeiro se destacou em relação à eficiência; embora com menos recursos, a utilização dos serviços foi relativamente mais elevada. O padrão observado parece refletir aspectos relacionados à regionalização e à trajetória política de cada assistência, o efeito silos. Os resultados podem subsidiar o desenho de políticas de saúde voltadas a superar o subdimensionamento da estrutura de serviços públicos assistenciais nas regiões de menor desenvolvimento socioeconômico e a busca de parâmetros e mecanismos de coordenação a fim de equilibrar indicadores de desempenho.

Palavras-chave Politica de saúde, Regionalização, Assistência odontológica, Assistência integral 


\section{Introdução}

No final da década de 1970, a descentralização foi uma das principais estratégias de transferência de poder e responsabilidades para os níveis locais no processo de reforma dos Estados nacionais na Europa em decorrência da crise econômica mundial e do esgotamento do Welfare State ${ }^{1}$. Na América Latina, a descentralização veio atrelada também a aspirações por democracia e expectativas positivas de superação dos problemas que os sistemas de saúde enfrentavam².

Enquanto em alguns países europeus a descentralização se articulou à regionalização, possibilitando a organização de redes de serviços associadas à criação e ao fortalecimento de autoridades sanitárias regionais ${ }^{3}$, na América Latina ambos os processos foram implementados com graus variados de articulação, sempre com maior destaque para a descentralização ${ }^{4}$. A oferta de serviços integrais que permitam a indivíduos e famílias obter o cuidado de que necessitam tem sido uma preocupação constante em todos esses processos.

No Brasil, a descentralização foi operacionalizada por meio da transferência de recursos e responsabilidades ao município, menor unidade da federação. Apesar de tal estratégia assegurar a autonomia da gestão local, a fim de proporcionar cuidado integral e equânime, no que diz respeito ao acesso universal ao sistema, houve um estrangulamento financeiro em decorrência de a maioria dos municípios não serem autossuficientes economicamente, mantendo sua dependência financeira em relação a estados e união ${ }^{6,7}$. Para enfrentar essa situação, estudos têm sugerido combinar a estratégia da regionalização à constituição das redes de atenção à saúde ${ }^{4,7-9}$.

Esse esforço entrou tardiamente na agenda política da saúde brasileira, apenas nos anos 2000, por meio de várias iniciativas, com a finalidade de garantir os princípios da universalização da saúde (integralidade, equidade), diminuir desigualdades socioespaciais, superar os limites da municipalização da saúde, fortalecer o papel dos estados no planejamento regional e propiciar maior segurança jurídica para as relações intergovernamentais de cooperação na construção das redes de atenção à saúde (RAS) ${ }^{10}$.

Construir redes que sejam de fato responsivas às necessidades da população significa assegurar, entre outros aspectos, a oferta de programas e serviços de saúde integrais, incluindo a assistência odontológica programática, cuja expansão passou a ser defendida no país a partir da $7^{\text {a }}$ Conferên- cia Nacional de Saúde, em 1980. Entretanto, até os anos 2000, a Política Nacional de Saúde Bucal (PNSB) tinha um caráter verticalizado e centralizador, que se expressava, entre outros aspectos, no predomínio de programas escolares e na oferta de consultas de urgência aos demais grupos populacionais. Em janeiro de 2004 foram aprovadas novas diretrizes para a política de saúde bucal no país $^{11}$, com o objetivo de ampliar o acesso à assistência odontológica e articular as ações de saúde bucal ao modelo de atenção integral à saúde ${ }^{12}$.

Considerando as desigualdades territoriais e a multiplicidade de atores governamentais e não governamentais, públicos e privados envolvidos na condução e na prestação da assistência à saúde, a crescente tensão entre as expectativas de atendimento da população e as limitações dos recursos disponíveis para o setor, a investigação da estrutura e do desempenho da assistência médica e odontológica em uma perspectiva regional pode ser útil para avaliar a adequação dos serviços e o nível de implementação da política de saúde. Ao contemplar um período mais largo, o estudo possibilita verificar a permanência ou alteração de padrões que podem refletir tendências da trajetória histórica ou flexões decorrentes de políticas públicas mais recentes.

A produção de informações científicas sobre a estrutura e o desempenho pode refletir padrões semelhantes ou distintos conforme o processo de implementação das RAS e a distribuição dos equipamentos de saúde nos territórios regionais. Tais padrões podem estar relacionados a múltiplos fatores, gerais e específicos.

Entre os aspectos gerais, destacam-se os impasses decorrentes das políticas de regionalização, como: a criação de redes de serviços baseadas em negociação, não em planejamento prévio; a atribuição de responsabilidades muito amplas para um nível de governo com capacidade limita$\mathrm{da}$; a falta de clareza nas regras; e lacunas no exercício de competências para o desenvolvimento das RAS nos territórios ${ }^{13}$.

Entre os aspectos específicos, diferenças decorrentes da trajetória política que tem marcado a assistência médica e a assistência odontológica podem estar associadas aos padrões de estrutura e desempenho. Alguns autores chamam atenção para o fenômeno da separação entre a assistência médica e a assistência odontológica ${ }^{14-17}$, um fato histórico e ao mesmo tempo um fator de dependência da trajetória política que muitos países experimentaram, incluindo o Brasil, e que poderia exercer importante influência sobre o grau de equivalência com que ambos os serviços se es- 


\section{Método}

Realizou-se um estudo de caso com abordagem qualitativa e quantitativa ${ }^{18}$. Para atender aos objetivos, duas regiões de saúde de condições sociais distintas foram selecionadas intencionalmente, tendo-se como ponto de partida a classificação formulada para as 438 regiões de saúde do país pela pesquisa "Política, Planejamento e Gestão das Regiões e Redes de Atenção à Saúde no Brasil"19.

De acordo com a situação socioeconômica e a oferta/complexidade dos serviços da saúde, as regiões de saúde brasileiras foram classificadas em cinco grupos: 1) baixo desenvolvimento socioeconômico e baixa oferta de serviços; 2) médio/ alto desenvolvimento socioeconômico e baixa oferta de serviços; 3 ) médio desenvolvimento socioeconômico e média oferta de serviços; 4) alto desenvolvimento socioeconômico e média oferta de serviços; e 5) alto desenvolvimento socioeconômico e alta oferta de serviços. Além disso, verificou-se o tipo de prestador predominante para a produção ambulatorial e as internações, cujas categorias eram: prestador predominantemente público; situação intermediária; e prestador predominantemente privado ${ }^{20}$.

As regiões escolhidas foram Juazeiro e Norte -Barretos (NB), por apresentarem características socioeconômicas e de oferta de serviços distintas entre si, estando posicionadas nos extremos dos grupos 1 e 5, respectivamente. Em ambas, o prestador era predominantemente privado.

A região Juazeiro, situada ao norte do estado da Bahia, é composta por dez municípios: Campo Alegre de Lourdes, Canudos, Casa Nova, Curaçá, Juazeiro, Pilão Arcado, Remanso, Sento Sé, Sobradinho e Uauá. Norte-Barretos, situada ao norte do estado de São Paulo, também apresenta dez municípios em sua região: Altair, Barretos, Cajobi, Colina, Colômbia, Guaíra, Guaraci, Jaborandi, Olímpia e Severínia.

A Tabela 1 apresenta os contrastes demográficos, socioeconômicos e da oferta dos serviços de saúde das regiões para anos selecionados.

A abordagem qualitativa envolveu pesquisa documental nos dossiês das respectivas regiões para extração das análises relativas às dimensões política, estrutural e organizacional do processo de regionalização em cada contexto. Os dossiês foram construídos a partir de pesquisa em campo, utilizando o instrumento da entrevista semiestruturada, com diversos atores (gestores e prestadores estaduais, regionais e municipais, além de representantes da sociedade por meio dos conselhos municipais de saúde). Para Norte -Barretos, foram realizadas 46 entrevistas, sendo 18 em Barretos, 8 em Olímpia e 7 em Cajobi. Em Juazeiro, foram realizadas 32 entrevistas, sendo 13 em Juazeiro, 11 em Remanso e 8 em Casa Nova.

Para a abordagem quantitativa foram utilizadas as seguintes fontes para a extração dos dados e a construção dos indicadores: Cadastro Nacional de Estabelecimentos de Saúde (CNES) e Sistema de Informação Ambulatorial (SIA), a partir de acesso ao Departamento de Informática do SUS (DataSUS). Foram analisados os anos de 2007 a 2014, pois constituem um período atravessado pela segunda e terceira fases do processo de regionalização, orientados, respectivamente, pelo Pacto pela Saúde e pela Contratualização. Além disso, os sete anos analisados nesta pesquisa atravessaram mais de uma gestão municipal em diferentes conjunturas políticas, permitindo a elaboração de proposições de caráter mais estrutural.

Os indicadores de estrutura tiveram por base alguns estudos ${ }^{21-25}$ e os números (n) de médicos e cirurgiões-dentistas (CD) por 10 mil habitantes que atendem no SUS. Além disso, foram apurados o número de cirurgiões-dentistas por 10 mil habitantes que atuam na Atenção Básica $(\mathrm{AB})$ e na Atenção Especializada (Centro de Especialidades Odontológicas - CEO), e também a porcentagem de população total potencialmente coberta pela Equipe de Saúde Bucal (ESB) da Estratégia de Saúde da Família (ESF).

Os indicadores de efetividade, que tiveram por base alguns estudos ${ }^{21,26}$, foram: números de consultas médicas na $\mathrm{AB}$, de procedimentos odontológicos básicos individuais e de procedimentos odontológicos especializados individuais, todos padronizados por 100 habitantes; cobertura de ação coletiva de escovação dental supervisionada para população de 5 a 14 anos; cobertura de primeira consulta odontológica programática; proporção de Equipe de Saúde Bucal (ESB) que ofertava conjunto mais abrangente de procedimentos na ESF; e proporção de 
Tabela 1. Indicadores demográficos, socioeconômicos e da oferta de serviços de saúde das regiões Norte-Barretos e Juazeiro em anos selecionados.

\begin{tabular}{|c|c|c|}
\hline \multirow{2}{*}{ Indicadores } & \multicolumn{2}{|c|}{ Região de Saúde } \\
\hline & Norte-Barretos & Juazeiro \\
\hline \multicolumn{3}{|l|}{ Demográficos e socioeconômicos } \\
\hline População total (2014) & 283.993 & 537.115 \\
\hline Número de municípios & 10 & 10 \\
\hline IDH (Aumento 2000-2010) & 0,687 a 0,824 & 0,506 a 0,677 \\
\hline $\begin{array}{l}\text { \% da população a partir de dez anos co } \\
\text { ensino fundamental (2010) }\end{array}$ & 52,9 & 33,6 \\
\hline \multicolumn{3}{|c|}{ \% da população a partir de dez anos com pelo menos ensino } \\
\hline \multicolumn{3}{|l|}{ PIB per capita $(\mathrm{R} \$)$} \\
\hline 2010 & $23.013,01$ & $6.884,75$ \\
\hline 2013 & $28.550,02$ & $8.461,60$ \\
\hline \multicolumn{3}{|l|}{ Renda domiciliar per capita ( $\mathrm{R} \$)$} \\
\hline 2000 & 657,97 & 235,07 \\
\hline 2010 & 805,72 & 331,90 \\
\hline \multicolumn{3}{|l|}{ Indicadores dos serviços de saúde } \\
\hline \multicolumn{3}{|c|}{ Internações de alta complexidade/100 mil habitantes } \\
\hline 2008 & 164 & 0,5 \\
\hline 2011 & 210 & 0,4 \\
\hline 2014 & 260 & 7,4 \\
\hline \multicolumn{3}{|l|}{ Quantidade de leitos/mil habitantes } \\
\hline 2007 & 4,0 & 2,0 \\
\hline 2011 & 3,2 & 2,0 \\
\hline 2014 & 3,3 & 1,7 \\
\hline \multicolumn{3}{|l|}{ Cobertura equipes de atenção básica (\%) } \\
\hline 2008 & 48,9 & 49,8 \\
\hline 2009 & 48,9 & 51,7 \\
\hline 2010 & 50,8 & 55,8 \\
\hline 2011 & 48,0 & 64,9 \\
\hline 2012 & 51,1 & 64,5 \\
\hline \multicolumn{3}{|l|}{ Equipamentos de saúde (2015) } \\
\hline UBS & 82 & 121 \\
\hline UBS com ESB mod. I & 33 & 86 \\
\hline CAPS & 3 & 10 \\
\hline CPN (Casa de Parto Natural) & - & 1 \\
\hline Hospital geral & 4 & 14 \\
\hline Hospital especializado & 8 & 3 \\
\hline Hospital dia & - & 3 \\
\hline Polo de academia da saúde & 6 & 4 \\
\hline UPA/PA & 6 & 1 \\
\hline SADT & 1 & 18 \\
\hline Unidade SAMU & 14 & 17 \\
\hline Equipamento diagnóstico por imagem & 278 & 106 \\
\hline Ambulatório de saúde do idoso & 2 & - \\
\hline Centro de testagem e aconselhamento & 3 & - \\
\hline Centro de reabilitação & 7 & - \\
\hline CEO & 3 & 2 \\
\hline
\end{tabular}

Fonte: IBGE, DataSUS/CNES. 
ESB que agenda consulta especializada atuando a favor da coordenação dos cuidados.

Os indicadores de eficiência apurados para cada ano do período tiveram por base alguns estudos $^{21,25,27}$ e foram: razão entre consultas médicas na $\mathrm{AB}$ e médicos que atendem na $\mathrm{AB}$ do SUS; razão entre o total de procedimentos de primeira consulta odontológica e quantidade de CD que trabalham na $\mathrm{AB}$ do SUS, razão entre a quantidade de procedimentos odontológicos básicos e $\mathrm{CD}$ que trabalham na $\mathrm{AB}$ do SUS; e razão entre a quantidade procedimentos odontológicos especializados e CD que trabalham no CEO.

Alguns indicadores apresentaram informações disponíveis a partir de 2008, pois nesse ano houve mudança na tabela de procedimentos do SUS, apresentando certa descontinuidade em relação aos anos anteriores. A interpretação dos dados se deu com o auxílio de planilhas criadas no programa Excel, além dos dados históricos e socioeconômico-epidemiológicos das regiões comparadas. Este estudo fez uso de dados secundários públicos. A pesquisa "Política, Planejamento e Gestão das Regiões e Redes de Atenção à Saúde no Brasil (Região e Redes)", a qual deu origem aos dossiês que são objeto deste estudo (aspectos qualitativos).

\section{Resultados}

No Quadro 1 são apresentados os resultados qualitativos presentes nos dossiês das respectivas regiões de estudo. O processo de regionalização tem sido reconhecido de forma positiva e a instância regional da esfera estadual tem tido grande importância para as duas regiões de saúde. Ambas entendem que a porta de entrada preferencial para a RAS deve ser a Unidade Básica de Saúde (UBS/ESF), compartilham a análise de que a capacidade instalada da RAS não tem sido adequada às necessidades de saúde da população, que o provimento da força de trabalho, especialmente da categoria médica, tem sido insuficiente e que a distribuição dos serviços não tem atendido à distribuição territorial da população, em decorrência da concentração de serviços em certos municípios, sobretudo nos municípios polos.

Algumas diferenças no âmbito da dimensão política se destacaram entre as regiões. Enquanto na região de melhor condição social os maiores protagonistas de conflitos têm sido os municípios e a instância regional, seguidos de municípios e governo estadual, na outra ponta os maiores protagonistas de conflitos têm sido os municípios e o governo estadual, seguidos de municípios e da instância regional. $\mathrm{Na}$ região Juazeiro a tomada de decisões é compartilhada com a região Petrolina, uma experiência inovadora, por meio do Colegiado Regional Interestadual (CRIE), cuja finalidade é discutir e pactuar decisões que envolvam a Rede de Saúde Pernambuco/Bahia.

No Quadro 2 são mostrados os resultados quantitativos, referentes aos indicadores de estrutura e desempenho (efetividade e eficiência) das respectivas regiões de estudo. Em relação à estrutura da assistência médica e odontológica, a razão médicos por habitante que atende no SUS em Norte-Barretos foi duas vezes maior que a razão dentistas por habitante, e três vezes maior para Juazeiro. Para a relação dentistas que trabalham no SUS que atendem na $\mathrm{AB}$ e atuam no CEO, a região Norte-Barretos foi melhor, variando de três a cinco vezes mais, a depender do indicador.

Para os indicadores de efetividade, Norte -Barretos foi melhor, com aumento da diferença entre as regiões. A cobertura média de ação coletiva de escovação dental supervisionada foi de duas a sete vezes maior do que a registrada na região Juazeiro.

Em relação à eficiência, Juazeiro apresentou valores mais elevados em todos os aspectos comparados. Para a assistência médica, por exemplo, o número de consultas na $\mathrm{AB}$ por profissional aumentou na região, enquanto em Norte-Barretos houve uma queda. Para o número de procedimentos especializados por profissional, a variação foi quase cinco vezes superior em Juazeiro.

\section{Discussão}

Aspectos estruturais e de desempenho da assistência médica e odontológica foram comparados em duas regiões do sistema de saúde brasileiro intencionalmente selecionadas em decorrência da elevada diferença nas condições socioeconômicas (PIB per capita, renda domiciliar per capita e escolaridade) e de oferta de serviços de saúde (internações de alta complexidade por mil habitantes e quantidade de leitos por mil habitantes). Os resultados mostraram um padrão misto, ou seja, ora favorável, ora desfavorável à região de melhores condições. Enquanto uma região se sobressaiu em relação aos indicadores de estrutura (exceto para cobertura estimada da ESB na ESF) e efetividade, a outra se destacou em quanto à eficiência, isto é, embora com menos recursos, a utilização dos serviços foi relativamente mais elevada. 
Quadro 1. Principais aspectos decorrentes da análise das dimensões política, organizacional e estrutural das regiões Norte-Barretos (2016) e Juazeiro (2017).

\begin{tabular}{|c|c|c|}
\hline $\begin{array}{l}\text { Dimensões } \\
\text { analisadas }\end{array}$ & Norte-Barretos & Juazeiro \\
\hline Política & $\begin{array}{l}\text { - Ordem de importância nas decisões sobre } \\
\text { saúde: Departamento Regional de Saúde } \\
\text { (DRS). } \\
\text { - Importância das instituições na tomada } \\
\text { de decisões sobre saúde: DRS > Ministério } \\
\text { Público > Secretaria Estadual de Saúde; } \\
\text { - Maiores protagonistas de conflitos: } \\
\text { municípios x instância regional > } \\
\text { municípios x governo estadual. }\end{array}$ & $\begin{array}{l}\text { - Ordem de importância nas decisões sobre } \\
\text { saúde: Colegiado Regional Interestadual. } \\
\text { - Importância das instituições na tomada } \\
\text { de decisões sobre saúde: Secretaria Estadual } \\
\text { de Saúde > conselhos participativos e poder } \\
\text { Legislativo > organização da sociedade civil } \\
\text { (mídia) > prestadores de serviços de saúde } \\
\text { (média e alta complexidade). } \\
\text { - Maiores protagonistas de conflitos: } \\
\text { municípios x governo estadual > municípios x } \\
\text { instância regional. }\end{array}$ \\
\hline Organizacional & $\begin{array}{l}\text { - Capacidade instalada da RAS não está } \\
\text { adequada às necessidades de saúde da } \\
\text { população. } \\
\text { - Há insuficiência de força de trabalho nas } \\
\text { regiões, especialmente da categoria médica. }\end{array}$ & $\begin{array}{l}\text { - Capacidade instalada da RAS não está } \\
\text { adequada às necessidades de saúde da } \\
\text { população. } \\
\text { - Há insuficiência de força de trabalho nas } \\
\text { regiões, especialmente da categoria médica. }\end{array}$ \\
\hline Estrutural & $\begin{array}{l}\text { - A distribuição dos serviços de saúde } \\
\text { não atende à distribuição territorial da } \\
\text { população. } \\
\text { - Há concentração de serviços nos } \\
\text { municípios, especialmente os municípios } \\
\text { polos. } \\
\text { - A porta de entrada para a RAS deve ser a } \\
\text { Unidade Básica de Saúde (UBS/ESF). }\end{array}$ & $\begin{array}{l}\text { - A distribuição dos serviços de saúde não } \\
\text { atende à distribuição territorial da população. } \\
\text { - Há concentração de serviços nos municípios, } \\
\text { especialmente os municípios polos. } \\
\text { - A porta de entrada para a RAS deve ser a } \\
\text { Unidade Básica de Saúde (UBS/ESF). }\end{array}$ \\
\hline
\end{tabular}

Fonte: Elaborado pelos autores com base em dados dos dossiês de pesquisa das regiões Norte-Barretos e Juazeiro.

Os resultados mostraram que a regionalização foi reconhecida de forma positiva em ambas as regiões por vários aspectos, entre os quais a potencial contribuição para a redução das desigualdades de acesso aos serviços, para a universalização da utilização da rede de saúde, para aumentar a possibilidade de diálogo e integração da rede e diminuir a chance de estrangulamento financeiro dos municípios. Ampliar o grau de compartilhamento e democratização dos problemas e das decisões, incluindo a distribuição dos recursos financeiros, é fundamental para que a regionalização seja construída de forma positiva e autônoma, pois o princípio da hierarquização é algo que encontra raízes no ordenamento jurídico do Estado brasileiro, de modo que, se não democratizada, a regionalização pode representar apenas mais uma forma de presença e controle das instituições estatais sobre o território ${ }^{28}$, suprimindo o protagonismo dos municípios que compõem e constroem o espaço geográfico. $\mathrm{Na}$ França, tem-se observado uma nítida tendência de democratização no nível regional do sistema de saúde ${ }^{29}$.
Foi comum entre as regiões a análise de que a distribuição dos serviços não tem atendido à demanda populacional, persistindo a concentração deles nos municípios polos. Examinando a extensão e os determinantes do processo de municipalização da política de saúde no Brasil, Arretche e Marques ${ }^{27}$ apontaram que quanto maior a população de um município, maior é a quantidade absoluta de equipamentos e serviços presentes sob a gestão municipal, sinal de ocorrência da municipalização.

Apesar da peregrinação do usuário pela rede de serviços ser um fator persistente, reconheceuse em ambas as regiões que a APS deveria ser a porta de entrada da RAS. A capacidade instalada não estava adequada às necessidades da população, especialmente em relação ao acesso a serviços de alta complexidade. Essa fragilidade de integração da rede assistencial persiste após a municipalização dos serviços de saúde e tem sido associada à dinâmica política do federalismo sanitário brasileiro. Há fragilidade nas relações cooperativas estabelecidas entre estados e municípios, dificultando as definições de atribuições 
Quadro 2. Indicadores de estrutura, efetividade e eficiência, regiões Norte-Barretos e Juazeiro, 2007-2014.

\begin{tabular}{|c|c|c|}
\hline Dimensão & Região Norte-Barretos & Região Juazeiro \\
\hline \multicolumn{3}{|c|}{ Estrutura } \\
\hline Número de médicos SUS/10 mil habitantes & Aumento de 15 para 25 & Aumento de 6,1 para 8,6 \\
\hline $\begin{array}{l}\text { Percentual da população potencialmente coberta pela } \\
\text { ESB pela ESF }\end{array}$ & $\begin{array}{l}\text { Aumento de } 29,7 \% \text { para } \\
37,1 \%\end{array}$ & $\begin{array}{l}\text { Aumento de } 38,1 \% \text { para } \\
52,6 \%\end{array}$ \\
\hline $\begin{array}{l}\text { Número de dentistas que atendem SUS/10 mil } \\
\text { habitantes }\end{array}$ & Variação: 6,9 e 6,4 & Variação: 1,8 e 2,4 \\
\hline Número de dentistas que atuam na $\mathrm{AB} / 10$ mil habitantes & Variação: 5,5 e 5,6 & Variação: 1,6 e 1,8 \\
\hline $\begin{array}{l}\text { Número de dentistas que atuam na assistência } \\
\text { especializada por } 10 \text { mil habitantes }\end{array}$ & Variação: 5,0 e 5,7 & Variação: 0,1 e 0,3 \\
\hline \multicolumn{3}{|c|}{ Efetividade } \\
\hline Número de consultas médicas na $\mathrm{AB} / 100$ habitantes & Variação: 12,4 e 14,5 & Variação: 2,4 e 7,3 \\
\hline $\begin{array}{l}\text { Cobertura na ação coletiva de escovação dental } \\
\text { supervisionada, população de } 5 \text { a } 14 \text { anos }\end{array}$ & Variação: 14,5 e $38,2 \%$ & Variação: 1,8 e $6,2 \%$ \\
\hline Cobertura primeira consulta odontológica programática & $\begin{array}{l}\text { Decréscimo de } 15 \% \\
\text { para } 10,4 \%\end{array}$ & $\begin{array}{l}\text { Decréscimo de } 22,8 \% \\
\text { para } 8,7 \%\end{array}$ \\
\hline $\begin{array}{l}\text { Proporção de ESB que ofertam conjunto mais } \\
\text { abrangente de procedimentos }\end{array}$ & $74,4 \%$ & $71 \%$ \\
\hline $\begin{array}{l}\text { Proporção de ESB que agendam consulta especializada } \\
\text { atuando a favor da coordenação dos cuidados }\end{array}$ & $86,6 \%$ & $93 \%$ \\
\hline $\begin{array}{l}\text { No de procedimentos odontológicos básicos } / 100 \\
\text { habitantes }\end{array}$ & $\begin{array}{l}\text { Decréscimo de } 44,8 \text { para } \\
41,6\end{array}$ & $\begin{array}{l}\text { Decréscimo de } 37,6 \text { para } \\
14,8\end{array}$ \\
\hline $\begin{array}{l}\text { No de procedimentos odontológicos especializados/100 } \\
\text { habitantes }\end{array}$ & $\begin{array}{l}\text { Aumento de } 15,1 \text { para } \\
25,3\end{array}$ & $\begin{array}{l}\text { Diminuição de } 7,7 \text { para } \\
4,7\end{array}$ \\
\hline \multicolumn{3}{|c|}{ Eficiência } \\
\hline $\begin{array}{l}\text { Razão anual entre consultas médicas } \mathrm{AB} / \text { médicos } \\
\text { atendem AB-SUS }\end{array}$ & $\begin{array}{l}\text { Diminuição de } 215 \text { para } \\
125\end{array}$ & Aumento de 186 para 196 \\
\hline $\begin{array}{l}\text { Razão anual entre procedimentos de primeira consulta } \\
\text { odontológica/dentistas que trabalham na } \mathrm{AB}\end{array}$ & $\begin{array}{l}\text { Diminuição de } 272 \text { para } \\
193\end{array}$ & $\begin{array}{l}\text { Diminuição de } 1758 \text { para } \\
500\end{array}$ \\
\hline $\begin{array}{l}\text { Razão anual entre procedimentos odontológicos } \\
\text { básicos/dentistas que trabalham na AB-SUS }\end{array}$ & Variação: 998,4 e 1.302,2 & Variação: 615,7 e 1.446,5 \\
\hline $\begin{array}{l}\text { Razão anual entre procedimentos especializados/ } \\
\text { dentistas que trabalham no CEO }\end{array}$ & Variação: 12,7 e 15,7 & Variação: 28,4 e 73,7 \\
\hline
\end{tabular}

Fonte: Elaborado pelos autores.

e responsabilidades, a despeito das tentativas de fortalecimento do papel dos estados na condução do SUS. Em algumas regiões nas quais a autonomia financeira dos municípios é maior, um tipo de federalismo mais cooperativo pode predominar, enquanto em outras cujos municípios detêm menor autonomia financeira um tipo de federalismo com forte dependência do ente federal pode estar presente ${ }^{30}$.

Com base nos indicadores, três pontos merecem destaque. Primeiro, a melhor estrutura e o maior volume absoluto de procedimentos na região mais favorecida em termos socioeconômicos e de oferta de serviços podem estar associados ao modelo excludente de desenvolvimento social e econômico do país, em que os direitos sociais foram expandidos por regimes autoritá- rios e orientados a produzir desigualdades entre categorias de cidadãos e entre diferentes regiões do país. O Sudeste, por exemplo, não concentrou apenas o crescimento do PIB, passou a contar também com uma infraestrutura de serviços urbanos mais ampla do que qualquer outra região. As regiões mais pobres, por sua vez, não eram apenas desprovidas de oportunidades de trabalho, mas também de todos os serviços básicos de infraestrutura residencial (água, esgoto, coleta de lixo $)^{31}$.

O segundo ponto diz respeito às desigualdades na estrutura da oferta de serviços, que tendem a se refletir em desigualdade de acesso. Populações com menor nível de escolaridade e renda tendem a acessar menos os serviços de saú$\mathrm{de}^{28}$. No Brasil, o efeito dessa desigualdade pode 
ser medido pela distribuição assimétrica do uso de serviços odontológicos pela população ${ }^{32}$.

O terceiro é o fato de uma força de trabalho menor realizar uma maior quantidade de procedimentos/ações de saúde bucal poder significar, de um lado, o subdimensionamento dos recursos odontológicos disponíveis, e de outro a sobrecarga de trabalho clínico/procedimental para os profissionais, sinalizando para uma possível lógica de modelo assistencial centrada na doença/procedimento (curativo) que reproduz uma orientação liberal-privatista ${ }^{33}$. Situações de subdimensionamento de recursos odontológicos não são incomuns no sistema de saúde brasilei$\mathrm{ro}^{23}$ e justificariam maior alocação de recursos e investimentos em educação permanente para reorientar de forma crítica o modelo de atenção e as estratégias de cuidado nas práticas dos serviços.

Como era esperado, a provisão de dentistas na assistência odontológica básica e especializada, vinculada ao SUS mostrou-se favorável à melhor região. A exceção foi a cobertura estimada da ESB pela ESF, que apresentou taxa mais elevada em Juazeiro. Cabe destacar também que as diferenças entre as regiões na provisão de dentistas vinculados ao SUS diminuíram no período em análise.

Com a PNSB, a atenção à saúde bucal passou a compor uma das prioridades no governo federal, impulsionando a implantação e expansão das ESB, especialmente na região Nordest $^{34-36}$. O Pacto pela Vida estabeleceu, para o biênio 20102011, meta de $40 \%$ de cobertura populacional por ESB, e as regiões Nordeste e Centro-Oeste conseguiram atingí-la ${ }^{35}$. Esse fato pode ajudar a explicar a maior expansão na região Juazeiro e indicar uma tendência a favor da equidade. Tal tendência foi observada também na implantação da Estratégia Saúde da Família, para a qual os incentivos federais têm sido mais vantajosos nas regiões de pior condição social ${ }^{36}$. Entre 2008 e 2012, a cobertura de equipes da $\mathrm{AB}$ na região Juazeiro foi maior em relação à região Norte-Barreto. Em 2011, por exemplo, a diferença a favor da região de pior condição social foi $16 \%$.

Quanto à estrutura da assistência médica, a razão médico/habitante foi maior na região de melhor condição social. À medida que o número de médicos que atendem no SUS aumentou ao longo da série histórica, a quantidade de internações por alta complexidade também aumentou. $\mathrm{O}$ fato de não diminuir o número de internações pode ter relação com a diminuição da quantidade de leitos hospitalares, resultado apontado em pesquisa realizada por Castro colaboradores ${ }^{21} \mathrm{e}$ também identificado no presente estudo. Destaca-se que a cidade de Barretos concentra um polo de referência nacional em tratamento para câncer, o que se reflete em uma demanda mais elevada e que requer maior número de médicos contratados em comparação a uma região que não tem o mesmo status.

$\mathrm{O}$ fato de alguns indicadores apresentarem um padrão diferente daquele que poderia ser esperado, tendo em vista as condições socioeconômicas e de oferta de serviços das regiões, sugere a presença de diferentes condicionantes, entre os quais pode-se destacar aqueles de caráter mais geral, atuando do plano federal para o plano local, e aqueles mais específicos, agindo do nível local para o geral. Entre os primeiros podem ser identificados o processo de regionalização e seus impasses ${ }^{13}$ e a PNSB que se dinamizou no período ${ }^{12}$. Entre as condicionantes de caráter mais local, podem ser relacionados, de um lado, o processo de ampliação da democracia sanitária por meio de diferentes canais de comunicação e espaços de articulação política em que regiões de maior privação social teriam mais possibilidades de vocalizarem suas necessidades, e de outro o efeito silos, traduzido pelas características particulares que têm marcado as políticas e práticas das diferentes modalidades de assistência, entre as quais a assistência médico-sanitária e a odontológica, cuja separação e isolamento foi descrita por vários pesquisadores ${ }^{14-17,37}$, um efeito que poderia representar forte dependência para a trajetória política da assistência odontológica e sua configuração em um sistema regional.

Mesmo com algumas orientações mínimas definidas e pactuadas, alguns impasses decorrentes da dinâmica política e particular de cada território parecem estar modulando a implementação das RAS. Um aspecto pode ser o predomínio da força de negociação e autonomia de certos municípios em detrimento de um processo de planejamento que favoreça uma perspectiva regional cooperativa de alocação de recursos. Têm sido observadas dificuldades em redistribuir esses serviços, seja por questões políticas (propaganda eleitoreira e barganhas) ou financeiras (o serviço pode garantir o repasse de algum recurso ao município). Outro aspecto pode ser a ampla responsabilização de um nível de governo com capacidade limitada, um contexto identificado mais claramente em uma das regiões sob estudo, que possui muitos municípios de pequeno porte com forte dependência financeira da União. Os resultados do estudo de caso mostraram que as dife- 
renças estruturais entre as regiões foram mantidas no período, o que permite sustentar a noção de que faltam instrumentos e mecanismos de indução da redução de desigualdades entre as regiões no processo de regionalização. E sua maioria, os conflitos protagonizados por municípios e estados (caso de Juazeiro) refletem, em alguma medida, o atravessamento de responsabilidades entre níveis de governo, sem levar em consideração a importância da instância regional. Além disso, as políticas não definem o financiamento das RAS regionais. A concepção de um orçamento global em nível regional (baseado na capitação, por exemplo) poderia ser uma estratégia ${ }^{13}$.

Outra proposição que pode estar associada ao padrão misto encontrado diz respeito ao efeito silos. A desvinculação entre a saúde bucal e a do restante do corpo foi construída nas formações médica e odontológica por gerações e se reflete até hoje $\mathrm{e}^{14}$. Como consequência, as modalidades de assistência à saúde atuam de modo separado, na forma de silos ${ }^{15-17,38}$, e isso poderia, em algum grau, explicar uma trajetória diversa do esperado, como foi observado neste estudo com relação ao desempenho.

O isolamento entre os cuidados de saúde geral e a assistência odontológica é reconhecido internacional e nacionalmente $e^{14-17}$, o que conduziu à necessidade de recomendações políticas para a vinculação dos serviços de saúde bucal à oferta dos cuidados à saúde geral ${ }^{16,38,39}$, pois ambas estão interrelacionadas, apesar de serem tratadas como independentes.

As diretrizes de políticas de saúde no Brasil também reconhecem a importância da integração entre a saúde bucal e os cuidados gerais de saúde. A integralidade é um princípio doutrinário do SUS e tem sido defendida como o eixo condutor dos processos de mudanças para uma ruptura de valores tradicionais na saúde, como a fragmentação da atenção e do cuidado às pesso$\mathrm{as}^{40}$. Uma estratégia para garantir tal princípio na prática foi a implantação das equipes multiprofissionais na ESF, incluindo as equipes de saúde bucal, bem como a orientação dessa estratégia (Atenção Básica/APS) como coordenadora dos cuidados para os demais níveis de atenção ${ }^{40,41}$. Em 2013, para cada duas equipes de saúde bucal havia três ESF no país ${ }^{35}$.

Integrar a saúde bucal à saúde geral não depende apenas da concepção conceitual da saúde, dos profissionais e dos cuidados que a envolvem. Significa também abordar o sistema de financiamento que viabiliza tanto as ações de saúde quanto aquelas específicas de saúde bucal ${ }^{37}$.
Implica compreender a evolução da política de assistência odontológica e identificar os desafios resultantes de sua separação do resto dos cuidados de saúde, explorando as consequências dessa divisão para o futuro da política de saúde bucal e da reforma do sistema de saúde ${ }^{37}$.

Em relação às limitações e alcances deste estudo, alguns aspectos precisam ser comentados. O registro das informações (SIA-SUS) que geraram o indicador de efetividade "Cobertura de ação coletiva de escovação dental supervisionada para a população de 5 a 14 anos" apresentou importantes oscilações nas séries históricas analisadas. Para minimizá-las, outliers foram detectados e removidos. Os indicadores selecionados para analisar a força de trabalho na assistência médica e odontológica devem ser considerados com cautela, pois utilizou-se apenas o filtro do CNES/ DataSUS, que pode apresentar problemas de sub ou sobre registro. A análise abrangendo um período largo, como a adotada no presente estudo, contribui para atenuar a instabilidade dos registros e pode subsidiar o desenho de políticas de saúde voltadas a superar o subdimensionamento da estrutura de serviços nas regiões de menor desenvolvimento socioeconômico e a busca de um maior equilíbrio nos indicadores de efetividade e eficiência entre as regiões de saúde. Aspectos relativos à orientação política dos partidos da coalizão responsável pela gestão do setor saúde de estados e municípios em cada região não foram analisados e poderiam trazer informações sobre o grau de competição ou de cooperação das estratégias de estruturação dos serviços ambulatoriais ${ }^{27}$. A continuidade da pesquisa com nova rodada de entrevistas e observações in loco poderia propiciar a investigação de detalhes relativos à utilização dos recursos disponíveis e às possibilidades de um rearranjo das atividades desenvolvidas dentro e fora das unidades de saúde, incluindo suas relações horizontais e verticais, a fim de atender a expectativas voltadas ao campo prático da organização dos serviços. Apesar do presente estudo de caso envolver enfoques incorporados (envolveu mais de uma unidade de análise), a generalização é um fator limitante deste método ${ }^{18}$.

Pode-se concluir que a estrutura e o desempenho da assistência médica e odontológica das duas regiões de saúde, distintas socioeconomicamente e na oferta de serviços, apresentaram um padrão misto, isto é, enquanto a região de melhor condição se sobressaiu em relação aos indicadores de estrutura (exceto para cobertura estimada da ESB na ESF) e de efetividade, a outra se destacou quanto à eficiência, ou seja, embora com 
menos recursos, a utilização dos serviços foi mais elevada. O padrão observado pode subsidiar o desenho de políticas de saúde voltadas a superar o subdimensionamento da estrutura de serviços públicos de assistência médica e odontológica nas regiões de menor desenvolvimento socioeconômico e a busca de parâmetros e mecanismos de coordenação visando um maior equilíbrio nos indicadores de efetividade e eficiência entre as regiões de saúde.

\section{Colaboradores}

JDB Carneiro contribuiu na concepção, no desenho do trabalho, na análise e interpretação dos dados, na revisão crítica do conteúdo e participou da versão final do manuscrito. Bousquat A contribuiu na revisão crítica do conteúdo e participou da versão final do manuscrito. SCL Chaves contribuiu na revisão crítica do conteúdo e participou da versão final do manuscrito. Frazão P contribuiu na concepção, no desenho do trabalho, na análise e interpretação dos dados, na revisão crítica do conteúdo e participou da versão final do manuscrito.

\section{Financiamento}

Ministério da Ciência, Tecnologia e Inovação (MCTI) e Ministério da Saúde (MS), por meio da Chamada MCTI/CNPq/CT - Saúde/MS/SCTIE/Decit no 41/2013, além de ter sido submetida ao Comitê de Ética da Faculdade de Medicina da Universidade de São Paulo. 


\section{Referências}

1. Pereira AMM, Lima LD, Machado CV, Freire JM. Descentralização e regionalização em saúde na Espanha: trajetórias, características e condicionantes. Saúde Debate 2015; 39(n. esp.):11-27.

2. Costa APC. Regionalização do Sistema Único de Saúde na Região de Juruá, Tarauacá/Envira, Acre, no início do século XXI [tese]. São Paulo: Faculdade de Saúde Pública; 2017.

3. Lima LD, Viana ALD, Machado CV, Albuquerque MV, Oliveira RG, Iozzi FL, Scatena JHG, Mello GA, Pereira AMM, Coelho APS. Regionalização e acesso à saúde nos estados brasileiros: condicionantes históricos e político-institucionais. Cien Saude Colet 2012; 17(11):2881-2892.

4. Viana ALD, Lima LD, Ferreira MP. Condicionantes estruturais da regionalização na saúde: tipologia dos Colegiados de Gestão Regional. Cien Saude Colet 2010; 15(5):2317-2326.

5. World Health Organization (WHO). Integrated health services - what and why? Geneva: WHO; 2008.

6. Viana ALD, Lima LD, Oliveira RG. Descentralização e federalismo: a política de saúde em novo contexto - lições do caso brasileiro. Cien Saude Colet 2002; 7(3):493-507.

7. Santos L, Campos GWS. SUS Brasil: a região de saúde como caminho. Saude Soc 2015; 24(2):438-446.

8. Lima LD, Viana ALD, Machado CV, Albuquerque MV, Oliveira RG, Iozzi FL, Scatena JHG, Mello GA, Pereira AMM, Coelho APS. Regionalização e acesso à saúde nos estados brasileiros: condicionantes históricos e político-institucionais. Cien Saude Colet 2012; 17(11):2881-2892.

9. Mello GA, Pereira ANCM, Uchimura LYT, Iozzi FL, Demarzo MMP, Viana ALD. O processo de regionalização no SUS: revisão sistemática. Cien Saude Colet 2017; 22(4):1291-1310.

10. Albuquerque MV, Viana ALD. Perspectivas de região e redes na política de saúde brasileira. Saúde Debate 2015; 39 (n. especial):28-38.

11. Brasil. Ministério da Saúde. Secretaria de Atenção à Saúde. Departamento de Atenção Básica. Coordenação Nacional de Saúde Bucal. Diretrizes da Política Nacional de Saúde Bucal. Brasília: MS; 2004.

12. Narvai PC, Frazão P. Saúde Bucal no Brasil: muito além do céu da boca. Rio de Janeiro: Editora Fiocruz; 2008.

13. Vargas I, Mogollón-Perez AS, Unger JP, da Silva MRF, De Paepe P, Vázques ML. Regional-based Integrated Healthcare Network policy in Brazil: from formulation to practice. Health Policy Plan 2014; 30(6):705717.

14. Botazzo C. Da arte dentária. São Paulo: Hucitec; 2000.

15. Hummel J, Phillips KE, Holt B, Hayes C. Oral health: an essential component of primary care. Qualis $\mathrm{He}$ alth; 2015.

16. Atchison KA, Weintraub JA, Rozier RG. Bridging the dental-medical divide. Case studies integrating oral health care and primary health care. J Am Dentl Assoc 2018; 149(10):850-858.

17. Watt RG, Daly B, Allison P, Macpherson LMD, Venturelli R, Listl S, Weyant RJ, Mathur MR, Guarnizo-Herreño CC, Celeste RK, Peres MA, Kearns C, Benzian H. Ending the neglect of global oral health: time for radical action. Lancet 2019; 394(10194):261-272.
18. Yin RK. Estudo de caso: planejamento e métodos. Porto Alegre: Bookman; 2001.

19. Viana ALD, Bousquat A, Pereira APCM, Uchimura LYT, Albuquerque MV, Mota PHS, Demarzo MMP, Ferreira MP. Tipologia das regiões de saúde: condicionantes estruturais para a regionalização no Brasil. Saude Soc 2015; 24(2):413-422.

20. Albuquerque MV, Viana ALD, Lima LD, Ferreira MP, Fusaro ER, Iozzi FL. Desigualdades regionais na saúde: mudanças observadas no Brasil de 2000 a 2016. Cien Saude Colet 2017; 22(4):1055-1064.

21. Castro ALB, Andrade CLT, Machado CV, Lima LD. Condições socioeconômicas, oferta de médicos e internações por condições sensíveis à APS em grandes municípios do Brasil. Cad Saude Publica 2015; 31(11):2353-2366.

22. Girardi S, Carvalho CL, Wan Der Maas L, Farah J, Freire JA. O trabalho precário em saúde: tendências e perspectivas na Estratégia da Saúde da Família. Divulgação em Saúde para Debate 2010; 45:11-23.

23. Jaccottet CMG, Barros AJD, Camargo MBJ, Cascaes AM. Avaliação das necessidades de tratamento odontológico e da capacidade produtiva da rede de atenção básica em saúde bucal no município de Pelotas, estado do Rio Grande do Sul, Brasil, 2009. Epidemiol Serv Saude 2012; 21(2):333-340.

24. Oliveira RS, Morais HMM, Goes PSA, Botazzo C, Magalhães BG. Relações contratuais e perfil dos cirurgiões-dentistas em centros de especialidades odontológicas de baixo e alto desempenho no Brasil. Saude Soc 2015; 24(3):792-802.

25. Cascaes AM, Dotto L, Bonfim RA. Tendências da força de trabalho de cirurgiões-dentistas no Brasil, no período de 2007 a 2014: estudo de séries temporais com dados do Cadastro Nacional de Estabelecimentos de Saúde. Epidemiol Serv Saude 2018; 27(1).

26. Fernandes JKB, Pinho JRO, Queiroz RCS, Thomaz EBAF. Avaliação dos indicadores de saúde bucal no Brasil: tendência pró-equidade? Cad Saude Publica 2016; 32(2):e00021115.

27. Arretche MTS, Marques E. Municipalização da saúde no Brasil: diferenças regionais, poder do voto e estratégias de governo. Cien Saude Colet 2002; 7(3):455479.

28. Guimarães RB. Regiões de saúde e escalas geográficas. Cad Saude Publica 2005; 21(4):1017-1025.

29. Cadeau E. Observações sobre os sentidos e a essência das experiências francesa e brasileira na área da democracia sanitária. Revista de Direito Sanitário 2004; $1(5)$.

30. Dourado DA, Elias PEM. Regionalização e dinâmica política do federalismo sanitário brasileiro. Rev Saude Publica 2011; 45(1):204-211.

31. Arretche MTS. Trajetória das desigualdades: como o Brasil mudou nos últimos cinquenta anos. São Paulo: Editora Unesp; 2015.

32. Moysés SJ. Desigualdades em saúde bucal e desenvolvimento humano: um ensaio em preto, branco e alguns tons de cinza. Revista Brasileira de Odontologia em Saúde Coletiva 2000; 1(1):7-17.

33. Narvai PC. Odontologia e saúde bucal coletiva. São Paulo: Editora Hucitec; 1994. 
34. Scarparo A, Zermiani TC, Ditterich RG, Pinto MHB. Impacto da Política Nacional de Saúde Bucal - Programa Brasil Sorridente - sobre a provisão de serviços odontológicos no Estado do Rio de Janeiro. Cad Saude Colet 2015; 23(4):409-415.

35. Pinho JRO, Souza TC, Vilas Bôas MD, Neves PAM. Evolução da cobertura das equipes de saúde bucal nas macrorregiões brasileiras. Rev Assoc Paul Cir Dent 2015; 69(1):80-85.

36. Pereira AMM, Castro ANB, Oviedo RAM, Barbosa LG, Gerassi CD, Giovanella L. Atenção primária à saúde na América do Sul em perspectiva comparada: mudanças e tendências. Saude Debate 2012; 36(94):482499.

37. Mertz EA. The dental-medical divide. Health Affairs 2016; 35(12):2168-2175.

38. Frenk J, Chen L, Bhutta ZA, Cohen J, Crisp N, Evans T, Fineberg H, Garcia P, Ke Y, Kelley P, Kistnasamy B, Meleis A, Naylor D, Pabloz-Mendez A, Reddy S, Scrimshaw S, Sepulveda J, Serwadda D, Zurayk H. Health professionals for a new century: transforming education to strengthen health systems in an interdependent world. Lancet 2010; 376(9756):1923-1258.

39. Pitts NB. NHS Dentistry: options for change in context - a personal overview of a landmark document and what it could mean for the future of dental services. BDJ Open 2003; 195(11):631-635.
40. Viegas SMS, Penna CMM. As dimensões da integralidade no cuidado em saúde no cotidiano da Estratégia de Saúde da Família no Vale do Jequitinhonha, MG, Brasil. Interface (Botucatu) 2015; 19(55):1089-1100.

41. Chaves SCL, Barros SG, Cruz DN, Figueiredo ACLF, Moura BLA, Cangussu MCT. Política Nacional de Saúde Bucal: fatores associados à integralidade do cuidado. Rev Saude Publica 2010; 44(6):1005-1013.

Artigo apresentado em 20/06/2019

Aprovado em 06/01/2020

Versão final apresentada em 08/01/2020

Editores-chefes: Romeu Gomes, Antônio Augusto Moura da Silva 